\title{
The Cost-Effectiveness of Oximetrix Pulmonary Artery Catheters in the Postoperative Care of Coronary Artery Bypass Graft Patients
}

\author{
Lawrence O. Larson, MD, and Jeffrey V. Kyff, MD
}

\begin{abstract}
As cost-containment pressures continue to escalate in the health care industry, the cost-effectiveness of new technologies must be verified if their use in the care of patients is to be justified. Oximetrix fiberoptic pulmonary artery catheters (Abbott Critical Care Systems, North Chicago, IL) are used for the continuous measurement of mixed venous oxygen saturation, and have been promoted as a cost-effective, early, and accurate indicator of hemodynamic changes in critically ill patients and patients undergoing major surgical procedures. This retrospective study analyzed two groups of patients
\end{abstract}

$\mathbf{T}$

HE CONTINUOUS measurement of mixed venous oxygen saturation $\left(\mathrm{S}_{\bar{v}} \mathrm{O}_{2}\right)$ by means of an Oximetrix pulmonary artery catheter (Abbott Critical Care Systems, North Chicago, IL) has been claimed to be an early, accurate, instantaneous, and cost-effective indicator of hemodynamic changes in critically ill patients. ${ }^{1-3}$ Differing opinions have been expressed regarding both the cost-effectiveness and usefulness of these catheters for the measurement of $\mathrm{SvO}_{2}$ in various classes of patients. ${ }^{2,3}$ Cost-containment pressures exerted upon hospitals and their medical staffs have been increasing for several years. Despite these pressures, costs continue to rise due to inflation, as well as to the introduction of new technologies such as the Oximetrix pulmonary artery catheter.

The cost of the Oximetrix pulmonary artery catheter purchased at the authors' institution is approximately four times that of a conventional pulmonary artery catheter. Since August 1985, Oximetrix pulmonary artery catheters

This article is accompanied by an editorial: Kaplan JA: Monitoring technology: Advances and restraints. J Cardiothorac Anesth 3:257-259, 1989

From the Department of Anesthesiology at the University of Michigan Medical Center, Ann Arbor.

Address reprint requests to Lawrence $O$. Larson, $M D$, Director of Cardiovascular Anesthesia, Sinai Hospital of Detroit, 6767 W Outer Drive, Detroit, MI 48235.

(c) 1989 by W.B. Saunders Company.

0888-6296/89/0303-0004\$03.00/0 undergoing routine, elective coronary artery bypass grafting. In the first group, fiberoptic catheters were not used, whereas these catheters were used routinely in the second group. Multiple variables were analyzed in each group in an attempt to document cost-effectiveness or salutary effect on outcome as a result of the use of these catheters. Neither could be confirmed. It was concluded that the use of Oximetrix pulmonary artery catheters is not cost-effective and had no effect on outcome in this group of patients.

() 1989 by W.B. Saunders Company.

have been routinely placed preoperatively in all patients undergoing elective coronary artery bypass grafting.

The purpose of this retrospective study was to determine the cost-effectiveness and possible alterations in postoperative care of coronary artery bypass graft patients following the introduction of pulmonary artery catheters with continuous measurement of $\mathrm{Sv}_{2}$. The cost-effectiveness of these catheters was evaluated by searching for a decrease in the various lengthof-stay parameters measured, or for a decrease in the number of postoperative days of mechanical ventilation or postoperative cardiac outputs performed in the group in which $\mathrm{SvO}_{2}$ was monitored. The effects of continuous $\mathrm{SvO}_{2}$ monitoring on hospital morbidity were evaluated by analysis of the length of the various postoperative stay parameters. Rates of mortality were also examined in these groups of patients during comparable periods before and after Oximetrix catheters were routinely used.

\section{MATERIALS AND METHODS}

The charts of 41 patients undergoing coronary artery bypass grafting by a single surgeon during the months of March and April, 1985 (group 1), and the charts of 46 patients undergoing the same procedure by the same surgeon during the same months in 1987 (group 2) were reviewed retrospectively. A retrospective methodology was the necessary mode of evaluation of the cost-effectiveness of this particular technological advance for two reasons. First, a restrospective audit ensured that no bias could be introduced into the study by knowledge on the part of physicians and other care providers that a study (particularly one that examined treatment and treatment decision-making) was 
Table 1. Variables That Indicate No Difference Between Groups

\begin{tabular}{lccc}
\hline \multicolumn{1}{c}{ Variable } & 1985 (Group 1) & 1987 (Group 2) & $P$ \\
\hline Total hospital days & $15.8 \pm 3.5$ & $13.2 \pm 2.3$ & 0.28 \\
Postoperative hospital days & $12.5 \pm 2.6$ & $11.5 \pm 2.2$ & 0.65 \\
Preoperative ABGs & $1.24 \pm 0.6$ & $0.28 \pm 0.4$ & 0.18 \\
Postoperative ABGs & $27.7 \pm 17.2$ & $26.9 \pm 11.8$ & 0.88 \\
Days of postoperative mechanical ventilation & $4.0+1.0$ & $3.5 \pm 0.6$ & 0.69 \\
Postoperative intensive care days & $6.8 \pm 1.9$ & $5.6 \pm 0.7$ & 0.42 \\
\hline
\end{tabular}

being conducted. Second, the surgeon involved felt so strongly that this monitoring technique was valuable that he opposed a randomized prospective study that would allocate patients into a group that would be denied, by any method, this form of monitoring.

The months of March and April of each year were chosen for study to assure that the house staff caring for these patients in the thoracic intensive care unit (ICU) would have a relatively high level of training and would not be new to either the institution or their training program. The same period in each year was chosen to assure that similar levels of training existed in the surgical house staff caring for the patients being studied. Patients operated upon by a single surgeon were chosen to eliminate the possibility that attitudes of the surgeon in charge might bias results. No protocol existed in the ICU governing the response to changes in measured $\mathrm{Sv}_{2}$. Decisions regarding these responses were solely at the discretion of the house officer on duty in the unit.

Variables were chosen for analysis in an attempt to highlight cost savings in the second group of patients, which might have been the result of monitoring of $\mathrm{S}_{\bar{v}} \mathrm{O}_{2}$ through the use of Oximetrix pulmonary artery catheters. The relative acuity of the two groups of patients and the differences in morbidity and mortality between these groups were also noted. Values for the following variables were determined for each patient: (1) total days of hospitalization, (2) total days of preoperative hospitalization, (3) total days of postoperative hospitalization, (4) number of preoperative arterial blood gas (ABG) determinations, (5) number of postoperative ABG determinations, (6) number of days of postoperative mechanical ventilation, (7) number of preoperative days spent in the ICU, (8) number of cardiac output determinations performed postoperatively, and (9) number of postoperative days spent in the ICU. Additionally, morbidity and mortality during the hospitalization were recorded for each group.

The mean value $\pm \mathrm{SD}$ for each variable was determined for each group. Comparisons between groups were then made for each variable using the Student's $t$-test. Differences between the two groups were considered significant if $P \leqq 0.05$

\section{RESULTS}

Data presented in Table 1 demonstrate no statistically significant difference between the two groups in the total number of hospital days, total number of postoperative hospital days, total number of preoperative ABG determinations, total number of postoperative ABG determinations, total number of days of postoperative mechanical ventilation, and total number of postoperative days spent in the ICU. In addition, no difference in the in-hospital morbidity or mortality rate was noted.

Group 1 had significantly longer preoperative hospitalizations $(P<0.01)$ and - a greater number of preoperative days spent in the ICU $(P<0.007)$. Group 2 had a greater number of postoperative cardiac output determinations $(P<0.02)$. Table 2 presents the data for the variables in which statistical significance was noted.

\section{DISCUSSION}

Hospitals, doctors, and the health care industry in general are presently caught in an ever more stringent cost-containment environment. As a result, technological advances in medical care can no longer be accepted for general use without convincing evidence of their cost-effectiveness, as well as their efficacy and safety. Cost-containment pressures are increasing from the federal sector as well as from private third-party payers. The proliferation of health maintenance organizations, preferred provider organizations, and other prepaid medical plans

Table 2. Variables That Indicate Differences Between Groups

\begin{tabular}{lccl}
\hline \multicolumn{1}{c}{ Variable } & 1985 (Group 1) & 1987 (Group 2) & $P$ \\
\hline Days of preoperative hospitalization & $3.3 \pm 0.4$ & $1.7 \pm 0.1$ & 0.01 \\
Preoperative intensive care days & $1.37 \pm 0.2$ & $0.11 \pm 0.1$ & 0.007 \\
Postoperative cardiac output determinations & $4.0 \pm 3.6$ & $13.9 \pm 10.0$ & 0.02 \\
\hline
\end{tabular}


has likewise increased the pressure for economy. Examples of governmental pressures applied to control the cost of medical care include regulations promulgated by the Health Care Financing Administration (HCFA) in implementing section 9.52 of the Omnibus Reconciliation Act of 1980. Section 127 of the Tax Equity and Financial Responsibility Act of 1982 (TEFRA) ${ }^{5}$ applied these regulations retroactively to December 1980 . In addition, the advent of the concept of diagnosis-related groups (DRGs) has further increased the pressures on hospitals, medical staff members, and others involved in the care of patients to reduce costs. When costs are not controlled, hospitals may be left liable for the difference between their actual costs and the established payment rate for any particular diagnosis. In this environment it becomes important to substantiate the cost-effectiveness of any new technology used in the care of patients. This is particularly true when the new technology costs more per patient to use, as is most often the case.

The lack of difference between the two groups regarding the variables displayed in Table 1 indicates that the overall level of acuity for the two groups of patients was comparable. The significantly longer preoperative hospitalizations and longer preoperative ICU stays of patients in group 1 may be interpreted as an indication of higher patient acuity in this group. However, it is thought that the lack of significant differences in other variables more specific for high patient acuity, such as total hospital days, total postoperative hospital days, total postoperative ICU days, total days of postoperative mechanical ventilation, and in-hospital mortality rates, more strongly supports the contention that the groups are comparable. The shorter preoperative hospitalization and preoperative ICU stay of group 2 patients is more likely the result of an increased demand for cost containment by decreasing resource utilization, ie, shorter hospital stays, in 1987.

The greater number of postoperative cardiac output determinations in group 2 may have been the result of continuous monitoring of $\mathrm{S}_{\bar{v}} \mathrm{O}_{2}$. Minute-to-minute changes in this value may have suggested to house staff physicians that changes in the patient's clinical status had occurred that warranted further investigation by cardiac output determination. No protocol existed in the ICU governing the response to changes in measured $\mathrm{S}_{\bar{v}} \mathrm{O}_{2}$. Due to a paucity of written information in patient charts, it was not possible, in this retrospective study, to determine clear indications for ordering cardiac output determinations or what changes in therapy, if any, were based on either the change in $\mathrm{S}_{\bar{v}} \mathrm{O}_{2}$ or the cardiac output. During March and April of 1985, cardiac output determinations in the ICU required that the respiratory therapy department be summoned to perform these tests; whereas during the 1987 period, these determinations were performed by the nursing staff in the ICU. Therefore, the greater number of postoperative cardiac output determinations may simply be a reflection of greater availability. Another consideration may be that the reported lack of correlation between $\mathrm{Sv}_{2}$ and cardiac output ${ }^{6,7}$ led the house staff to obtain a greater number of cardiac output determinations. Changes in $\mathrm{S} \bar{v} \mathrm{O}_{2}$ may be brought about by changes in cardiac output, hemoglobin concentration, oxygen delivery, and oxygen consumption. ${ }^{8}$ This requires that a change in $\mathrm{S}_{\bar{v}} \mathrm{O}_{2}$ be evaluated to determine its etiology. Usually, this means obtaining the value of the cardiac output and other parameters of hemodynamic function. ${ }^{9}$ Of interest is a similar increase in the number of cardiac output determinations reported by Pearson et al (personal communication, January 1988) in their study of the cost-effectiveness of Oximetrix pulmonary artery catheters in the treatment of patients following all types of open heart surgery. They attributed this increase in the number of cardiac output determinations in their study to possible overemphasis on the importance of monitored changes in $\mathrm{S}_{\bar{v}} \mathrm{O}_{2}$.

It could be argued that the greater number of cardiac output determinations performed in group 2 reflected a superior level of care that was much more attuned to minute-to-minute variations in patient condition. Mitigating against this argument is the lack of difference in those parameters listed in Table 1. A superior level of patient care would be expected to resull in shorter ICU and hospital stays, shorter periods of mechanical ventilation, fewer ABG determinations, and improved mortality in group 2 . There is also no indication that the fewer number of preoperative hospital and ICU days in group 2 
were linked to the use of the fiberoptic pulmonary artery catheter. Forty-three out of 46 , or $93.5 \%$, of these catheters were placed as a routine measure immediately before surgery and were not used preoperatively in the ICU.

This study provides no evidence that the use of Oximetrix pulmonary artery catheters for the measurement of $\mathrm{S}_{\bar{v}} \mathrm{O}_{2}$ in the population studied has any cost-containment influence. In fact, the study suggests that the postoperative measurement of $\mathrm{Sv}_{2}$ may increase the cost of care in this group by requiring more frequent cardiac output determinations during the postoperative period. The increased annual cost of routine use of Oximetrix pulmonary artery catheters in coronary artery bypass graft patients in the study institution is approximately $\$ 71,500$ on a projected annual caseload of 550. The cost of the increased number of cardiac output determinations in the 1987 group cannot be calculated because their cost was included in the basic daily intensive care charge. This daily charge was $\$ 114$ greater in the 1987 group, but no mechanism exists in the institution to separate the portion of this charge attributable to cardiac outputs. This study does not directly address the issue of increased quality of care given patients as a result of utilization of Oximetrix pulmonary artery catheters to constantly measure $\mathrm{S}^{-} \mathrm{O}_{2}$. Indirect evidence that the quality of care in this group was not significantly improved is demonstrated by the lack of difference in postoperative hospital days, postoperative ABG determinations, days spent on mechanical ventilation postoperatively, and overall mortality. It would appear, based on the data, that the increased expense incurred by the use of Oximetrix pulmonary artery catheters for the continuous measurement of $\mathrm{SvO}_{2}$ in patients undergoing elective coronary artery bypass grafting cannot be justified.

\section{REFERENCES}

1. Gore JM, Sloan K: Use of continuous monitoring of mixed venous saturation in the coronary care unit. Chest 86:757-761, 1984

2. Norfleet EA, Watson CB: Continuous mixed venous oxygen saturation measurement: A significant advance in hemodynamic monitoring? J Clin Monit 1:245258,1985

3. Waller JL, Kaplan JA, Bauman DI, et al: Clinical evaluation of a new fiberoptic catheter oximeter during cardiac surgery. Anesth Analg 61:676-679, 1982

4. Public Law 96-49: 42 CFR Part 420, pub at Fed Reg 58260, Dec 30, 1982

5. Public Law 97-248: TEFRA Sec 127, pub at Fed Reg 43282, Sept 30, 1982
6. Kyff JV, Vaughn S, Yang SC, et al: Continuous monitoring of mixed venous oxygen saturation in patients with acute myocardial infarction. Chest (in press)

7. Schmidt CR, Frank LP, Forsythe SB, et al: Continuous $\mathrm{S}_{\overline{\mathrm{O}}} \mathrm{O}_{2}$ measurement and oxygen transport patterns in cardiac surgery patients. Crit Care Med 12:523, 1984

8. Aberman A: Fundamentals of oxygen transport physiology in a hemodynamic monitoring context, in Schweiss JF (ed): Continuous Measurement of Blood Oxygen Saturation in the High-Risk Patient. San Diego, Beach International, 1982, pp 13-26

9. Puri VK, Kyff JV: Pulmonary artery catheterization, uses and abuses. Int $\mathrm{J}$ Clin Monit Comput 3:107-115, 1986 